\title{
Audiometric Comparison Between the First Patients With the Transcutaneous Bone Conduction Implant and Matched Percutaneous Bone Anchored Hearing Device Users
}

\author{
*Cristina Rigato, *Sabine Reinfeldt, *Bo Håkansson, *Karl-Johan Fredén Jansson, \\ $\dagger$ Myrthe K. S. Hol, and †Måns Eeg-Olofsson \\ * Division of Signal Processing and Biomedical Engineering, Department of Signals and Systems, Chalmers University of Technology, \\ Gothenburg, Sweden; †Department of Otorhinolaryngology, Radboud University Medical Centre, Nijmegen, The Netherlands; and \\ tDepartment of Otorhinolaryngology, Head and Neck Surgery, Sahlgrenska University Hospital, The Sahlgrenska Academy, \\ University of Gothenburg, Gothenburg, Sweden
}

Hypothesis: The transcutaneous bone conduction implant (BCI) is compared with bone-anchored hearing aids (BAHAs) under the hypothesis that the BCI can give similar rehabilitation from an audiological as well as patient-related point of view.

Background: Patients suffering from conductive and mixed hearing losses can often benefit more from rehabilitation using bone conduction devices (BCDs) rather than conventional air conduction devices. The most widely used $\mathrm{BCD}$ is the percutaneous BAHA, with a permanent skin-penetrating abutment. To overcome issues related to percutaneous BCDs, the trend today is to develop transcutaneous devices, with intact skin. The BCI is an active transcutaneous device currently in a clinical trial phase. A potential limitation of active transcutaneous devices is the loss of power in the induction link over the skin. To address this issue, countermeasures are taken in the design of the BCI, which is therefore expected to be as effective as percutaneous BCDs. Methods: An early observational study with a matched-pair design was performed to compare $\mathrm{BCI}$ and BAHA groups of patients over several audiometric measurements, including speech audiometry and warble tones thresholds with and without the device. Additionally, questionnaires were used to assess the general health condition, benefit, and satisfaction level of patients.

Results: No statistically significant difference was detected in any of the audiological measurements. The outcome of patient-related measurements was slightly superior for BCI in all subscales.

Conclusion: Results confirm the initial hypothesis of the study: the BCI seems to be capable of providing as good rehabilitation as percutaneous devices for indicated patients. Key Words: Active transcutaneous-Audiometric measurements-Bone anchored hearing aid-Bone conduction hearing-Bone conduction implant-Hearing devicesQuestionnaires.

Otol Neurotol 37:1381-1387, 2016.
Patients suffering from conductive or mixed hearing losses are sometimes unable to use conventional air conduction hearing aids for various reasons. In many cases, bone conduction devices (BCDs) are an effective

Address correspondence and reprint requests to Cristina Rigato, M.Sc., Department of Signals and Systems, Chalmers University of Technology, SE-412 96 Gothenburg, Sweden;

E-mail: rigato@chalmers.se

The co-author B.H. holds several patents related to the $\mathrm{BCI}$ device. Together with co-authors S.R. and M.E.-O., he acts as a consultant for Oticon Medical.

The authors disclose no conflicts of interest.

This is an open-access article distributed under the terms of the Creative Commons Attribution-Non Commercial-No Derivatives License 4.0, where it is permissible to download and share the work provided it is properly cited. The work cannot be changed in any way or used commercially.

DOI: $10.1097 / \mathrm{MAO} .0000000000001183$ solution for rehabilitation of this patient group. The percutaneous bone anchored hearing aid (BAHA) has been the most widely used BCD with several years of satisfactory outcomes in both children and adults (1-3). New surgical techniques and abutment designs have been developed over the years to reduce clinical complications such as skin overgrowth around the abutment, infection, necrosis, and implant loss (4-6). Nevertheless, skinpenetration-related medical issues, together with a desire for aesthetical improvement, have led the development toward transcutaneous devices (7).

In the current study, two BCDs belonging to different categories, namely percutaneous and active transcutaneous, are compared. The first is a BAHA where the audio processor unit comprising a transducer is snapped onto an abutment that penetrates the skin and is anchored directly in the skull bone. The second is the 
bone conduction implant $(\mathrm{BCI})$, an active transcutaneous device where the transducer is implanted with flat surface contact in the temporal bone and the external audio processor is magnetically retained on the skin (8). The sound signal is transmitted electromagnetically by an inductive link through the skin. The main difference between the two devices is thus the soft tissue penetration with the BAHA, and intact skin with the BCI.

Today, hearing rehabilitation provided by BAHAs is generally successful and the development is more focused on the titanium fixture and abutment design and on aesthetic related issues. With an inductive link, problems related to the percutaneous solution are avoided, but with the risk of losing transmission efficiency. Two factors in the BCI are balancing out this loss in hearing sensitivity: 1) the attachment position of the $\mathrm{BCI}$ transducer, closer to the cochlea compared with a BAHA, has been shown to enhance the hearing sensitivity by 3 to $14 \mathrm{~dB}$ in the frequency range 125 to $8000 \mathrm{~Hz}$ $(9,10)$, and 2) there is a high-frequency boost in the transducer to increase the output force around 2500 to $6500 \mathrm{~Hz}$ in the frequency response (11). Considering these features, the $\mathrm{BCI}$ is expected to be as powerful as percutaneous devices.

Comparative studies are gaining more and more importance as the variety of BCDs is rapidly increasing. The success of rehabilitation provided by a hearing device is strongly dependent on the features of the device itself in combination with the patient's hearing characteristics and personal needs. It is therefore important to consider technical, audiometric, and subjective aspects when prescribing a specific device to a patient. Comparative studies are used to determine how different devices affect the individual rehabilitation of patients with similar hearing condition. In the literature, it is common to find comparative studies where the devices are fitted to the same patient $(12-15)$, or retrospective studies $(16,17)$. For implanted $\mathrm{BCDs}$, an alternative method is to select two patients who are using different devices but have similar hearing loss, and compare the rehabilitation provided by their respective devices. With such an approach, the current study is the first of its kind, allowing active transcutaneous and percutaneous devices to be compared in their proper utilization, i.e., implanted and on abutment.

This is an observational study that aims at comparing audiological and patient-related performance of the BCI with BAHAs under the hypothesis that they can provide similar rehabilitation.

\section{METHODS}

The study was approved by the Regional Ethical Review Board in November 2014.

\section{Hearing Devices}

Two devices, the BCI and a BAHA, were compared.

The $\mathrm{BCI}$ is an active transcutaneous device currently in the phase of long-term clinical trials $(8,18,19)$. The inclusion criteria is a bone conduction $\mathrm{PTA}_{4}$ (pure-tone average at $500,1000,2000$, and $4000 \mathrm{~Hz}$ ) of $30 \mathrm{~dB} \mathrm{HL}$ or better, and an average air-bone gap of at least $20 \mathrm{~dB}$. For the BCI device used in this study, the development of the signal processing software is still at an initial phase and no automatic functions have been implemented yet.

For the audiological comparison, a Ponto Pro Power (Oticon Medical, Askim, Sweden) was used. It is a BAHA designed to fit patients with maximum average bone conduction threshold of $55 \mathrm{~dB} \mathrm{HL}$ calculated at $500,1000,2000$, and $3000 \mathrm{~Hz}$. The device was fitted before measurements using Oticon software Genie Medical 2011.1 performing in situ audiometry and disabling all automatic functions. For the subjective outcome, the BAHA patients used their own device listed in Table 1.

\section{Participant Selection}

The first six patients operated for BCI and six patients using BAHA were included in the study. Each BAHA user was matched to one BCI user, resulting in six matched pairs. BAHA users were selected based on hearing, sex, and age characteristics. For each pair, the matching criteria were set as follows: I) PTA $_{4}$ of the ipsilateral ear: maximum $15 \mathrm{~dB}$ difference, for both bone and air conduction thresholds; II) sex: same; III) age: maximum $10 \%$ difference.

Criteria II and III had to be disregarded in one case each, due to difficulties in recruiting patients. Details about the participants can be found in Table 1. All participants are considered experienced users, with 6-month wearing time for the BCI patients and average of 9 years for the BAHA users (for details, see Table 1).

TABLE 1. Participants' age, sex, pure-tone hearing threshold average at 500, 1000, 2000, and 4000 Hz (PTA $)$ for air and bone conducted sound (AC and $B C$ ), model of utilized device and time from device implantation to present

\begin{tabular}{|c|c|c|c|c|c|c|}
\hline $\begin{array}{l}\text { Matched Pair } \\
\text { ID BCI/BAHA }\end{array}$ & $\begin{array}{c}\text { Age at } \\
\text { Test Date (Yr) } \\
\text { BCI/BAHA }\end{array}$ & $\begin{array}{c}\text { Sex } \\
\text { BCI/BAHA }\end{array}$ & $\begin{array}{c}\mathrm{PTA}_{4} \mathrm{AC}(\mathrm{dB} H \mathrm{HL}) \\
\mathrm{BCI} / \mathrm{BAHA}\end{array}$ & $\begin{array}{c}\mathrm{PTA}_{4} \mathrm{BC}(\mathrm{dB} \mathrm{HL}) \\
\text { BCI/BAHA }\end{array}$ & Own BAHA Device & $\begin{array}{c}\text { Time From } \\
\text { Device Implantation } \\
\text { BCI/BAHA }\end{array}$ \\
\hline 1 & $42 / 44$ & $\mathrm{~F} / \mathrm{F}$ & $65.0 / 75.0$ & $20.0 / 27.5$ & Cochlear Baha 4 & $6 \mathrm{~m} / 5 \mathrm{y}$ \\
\hline 2 & $49 / 53$ & $\mathrm{M} / \mathrm{F}(*)$ & $52.5 / 52.5$ & $30.0 / 26.3$ & Cochlear Baha Compact & $6 \mathrm{~m} / 7 \mathrm{y}$ \\
\hline 3 & $18 / 24(*)$ & $\mathrm{M} / \mathrm{M}$ & $53.8 / 60.0$ & $-3.8 / 3.8$ & Cochlear Baha Compact & $6 \mathrm{~m} / 4 \mathrm{y}$ \\
\hline 4 & $67 / 73$ & $\mathrm{~F} / \mathrm{F}$ & $56.3 / 68.8$ & $12.5 / 23.8$ & Oticon Medical Ponto Pro & $6 \mathrm{~m} / 13 \mathrm{y}$ \\
\hline 5 & $48 / 52$ & $\mathrm{~F} / \mathrm{F}$ & $73.8 / 65.0$ & $30.0 / 25.0$ & Cochlear BP100 & $6 \mathrm{~m} / 13 \mathrm{y}$ \\
\hline 6 & $49 / 45$ & $\mathrm{M} / \mathrm{M}$ & $67.5 / 70.0$ & $15 / 23.8$ & Cochlear BP100 & $6 \mathrm{~m} / 13 \mathrm{y}$ \\
\hline
\end{tabular}

The $\left(^{*}\right)$ indicates an exception in the matching criteria, i.e., age exceeding $10 \%$ deviation in pair 3 and different sex in pair 2 . BAHA indicates bone anchored hearing aid; BCI, bone conduction implant; F, female sex; M, male; m, months; y, years. 
Taking part in the study was voluntary and no remuneration was given to any of the participants.

\section{Audiometric Measurements}

Audiometric measurements with the BCI had previously been performed as a part of the clinical study and are reported in (18).

Audiometric measurements with the BAHA were carried out applying the same device on each patient's abutment. The sequence of measurements in each session was set in a random way to avoid systematic errors. After fitting the BAHA, no fine tuning was allowed even if in two cases the patients claimed that the setting was louder than needed.

All the audiometric measurements took place in a sound proof room of $16 \mathrm{~m}^{3}$. Speech material was played from CDs "Tal i brus" and "Svensk Talaudiometri'" in the patients' native language (Swedish), while tones were played directly from the clinical audiometer AC40 (Interacoustics A/S, Assens, Denmark). The sound source was a set of two loudspeakers positioned approximately $1 \mathrm{~m}$ in front of the patient seated in the middle of the room. When the contralateral ear hearing level was superior or similar to the tested ear, the nontest ear was blocked during all measurements to remove the air-conducted sound. The blocking was done by inserting an ear-plug (E-A-R Classic Soft) when anatomically feasible and by covering the ear with an earmuff (Peltor $3 \mathrm{M}$ Svenska AB, Sollentuna, Sweden). The plug was inserted deep into the ear canal to avoid the occlusion effect (20).

1. Sound Field Warble Tone Thresholds

Tone threshold audiometry was performed to measure the individual's hearing sensitivity for calibrated tones from 250 to $8000 \mathrm{~Hz}$.

The use of a warble tone was preferred to a continuous one to reduce the risk of standing waves in the test room and to allow patients potentially with tinnitus to distinguish the test signal from the noise (21).

The threshold was determined by the ascending procedure described in standard ISO 8253-1 (22).

2. Speech Recognition Score

Measurements of speech recognition score (SRS) in sound field were carried out according to standard ISO 8253-3 (23). A list of 50 mono-syllabic phonetically balanced words was presented at a fixed level of $63 \mathrm{~dB}$ SPL with superimposed low noise ( $4 \mathrm{~dB}$ signal-to-noise ratio). The SRS index was calculated as the percentage of correctly repeated words.

3. Speech Recognition Threshold in Quiet

The procedure described in standard ISO 8253-3 (23) was followed for the sound field speech recognition threshold (SRT) measurements. The listener was required to recognize spondaic words and the threshold was calculated as the level at which $50 \%$ of the words were correctly detected.

4. Signal-to-Noise Ratio Threshold

The sound field signal-to-noise-ratio (SNR) threshold is the threshold that results in 50\% speech intelligibility. The speech material used in this test has been developed and evaluated by Hagerman $(24,25)$. It consists of a list of 10 five-words sentences played together with a continuous noise with the same spectral characteristics as the speech. The speech was kept constant at conversational level (63 dB SPL), while the noise level was changed according to the adaptive method described by
Hagerman (26). The test was carried out only for the aided condition since unaided measurements would have required a higher speech level, making the aided and unaided thresholds incomparable.

\section{Questionnaires}

Two questionnaires were completed by each patient referring to the experience with their own hearing device.

1. Abbreviated Profile of Hearing Aid Benefit (APHAB) The questionnaire, developed by Cox and Alexander (27), consists of 24 statements rated with a 7-item scale with the score finally transposed into a percentage.

Four subscales are included in the questionnaire: 1) ease of communication (EC), regarding communication under favorable conditions; 2) reverberation (RV), focusing on sound perception in reverberant environments; 3) background noise ( $\mathrm{BN})$, dealing with noise interfering with the communication; and 4) aversiveness (AV) of sound, regarding the reaction to loud or unpleasant sounds.

2. Glasgow Benefit Inventory (GBI)

This questionnaire, described by Robinson et al. (28), is a postinterventional measure of the patient's benefit developed specifically for ear, nose, and throat interventions. The 18 questions of GBI aim at evaluating the change in health status as a whole, including psychological, social, and physical aspects.

The score is divided into three subscales investigating general changes in health status (GSS), social support (SSS), and physical health status (PHS). Questions are answered according to a 5-item Likert scale and the final score is expressed on a scale from -100 to +100 , where -100 corresponds to maximal negative benefit and +100 to maximal positive benefit (28).

\section{Statistical Analysis}

The relevance of improvement given by each device relative to the unaided condition was tested using the Wilcoxon signed rank test, with the null hypothesis that the median difference between aided and unaided conditions was zero. The same test was applied to compare the improvement given by different devices on matched pairs, assuming that the matching of patients ensures the applicability of this test. Data analysis was performed with Microsoft Excel (Microsoft Corporation, Washington, U.S.A.) and MatLab (MathWorks, Inc., Natick, MA, U.S.A.).

\section{RESULTS}

\section{Audiometric Measurements}

Compared with the unaided condition, both devices gave significant improvement over the whole frequency range, according to Wilcoxon signed rank test. As shown in Figure 1, the average improvement was maximum $41.0 \mathrm{~dB}$ with the $\mathrm{BCI}$ at $750 \mathrm{~Hz}$ and $35.0 \mathrm{~dB}$ with the BAHA at $1000 \mathrm{~Hz}$. The BCI performed slightly better in the mid-frequencies, while the BAHA had superior results for the lowest and the highest frequencies. The $\mathrm{PTA}_{4}$ improvement was $31.0 \pm 8.0 \mathrm{~dB}$ for the $\mathrm{BCI}$, and $31.5 \pm 2.8 \mathrm{~dB}$ for the BAHA. There was no statistically significant difference between the two devices. 
As can be observed in Figure 2A, the SRS in noise raised from an average of $4.5 \%$ (unaided condition) to $55.7 \%$ aided with the BCI, and from 7.7 to $59.3 \%$ with the BAHA. Average SRS was thus improved by $51.2 \pm 8.9 \%$ with the BCI, and $51.6 \pm 18.0 \%$ with the BAHA. The improvement relative to the unaided condition was statistically significant in both cases, but no difference could be detected between the two devices.

In Figure 2B, the SRT results are shown. In the BCI group, the SRT was decreased from 77.0 to $50.0 \mathrm{~dB} \mathrm{HL}$, and from 75.7 to $47.8 \mathrm{~dB} \mathrm{HL}$ with the BAHA. The improvement over the unaided condition $(27.0 \pm 7.6$ and $27.9 \pm 5.0 \mathrm{~dB}$ for the $\mathrm{BCI}$ and the BAHA, respectively) was again significant in both cases, but not the difference between the two devices.

The SNR-threshold, Figure 2C, was found to be $-5.5 \pm 2.3 \mathrm{~dB}$ for the BCI, practically the same as for the BAHA, $-5.7 \pm 1.9 \mathrm{~dB}$.

\section{Questionnaire Results}

The subjective comparison is between the BCI and different BAHAs rather than between two specific devices. In Figure 3, APHAB results are expressed as a percentage of improvement, obtained as the difference between the percentage of problems experienced in unaided condition and while using the device. In everyday life situations, the BCI obtained better rating than the BAHAs: EC $66.7 \%$ versus $25.7 \%$, BN $52 \%$ versus $42.5 \%$, RV $67.5 \%$ versus $38.2 \%$. Both groups reported percentages below zero in AV subscale, $-36.3 \%$ and $-23.6 \%$ for BCI and BAHA, respectively. Improvements in the first three subscales are significant according to the criteria given by Cox et al. (28). Significant differences between the two groups were found by Wilcoxon signed rank test for EC and RV subscales.

Results from the GBI questionnaire for both patient groups (Figure 3) showed significant improvement for the total score as well as for the GSS. The total score was slightly superior for BCI users compared with BAHA users, with an improvement of 36.2 and 32.0 points respectively, although not statistically significant. Approximately, the same score was achieved in the GSS (BCI: +50, BAHA: +49 points), while higher result was achieved by the BCI group in the SSS $(+19.4$ vs +2.8 points). In the PHS subscale BCI users reported an average improvement of 5.6 points, while the BAHA users reported a negative average score of -8.3 points.

\section{DISCUSSION}

\section{Study Design}

The design of the presented study is unique in the hearing aid research field as the two devices were tested on matched pairs of patients, whereas the closest design in a previous study included matching on a group level $(29,30)$. The matched-pairs structure of the study, although still exploratory, allows a valuable comparison of the devices despite the low number of participants. With only six patients included so far in the BCI clinical study, a randomized choice of six BAHA users was not advisable as the risk was to have a population sample very different from the BCI users group because of different inclusion criteria for the two BCDs. Furthermore, the six BCI patients had very diverse audiological characteristics and it was considered important to keep the same variability in the BAHA group.

Criteria for selection of patients were thus set both on hearing characteristics and on personal details. No constraint was set on the contralateral ear as the nontest side was blocked during all audiological measurements and the analysis was limited to the implanted-side ear. Contralateral hearing characteristics might play a more important role in the questionnaires outcome when evaluating the unaided condition, but the effect is mitigated by carrying out the analysis in a relative perspective, i.e., by looking at the difference between the aided and the

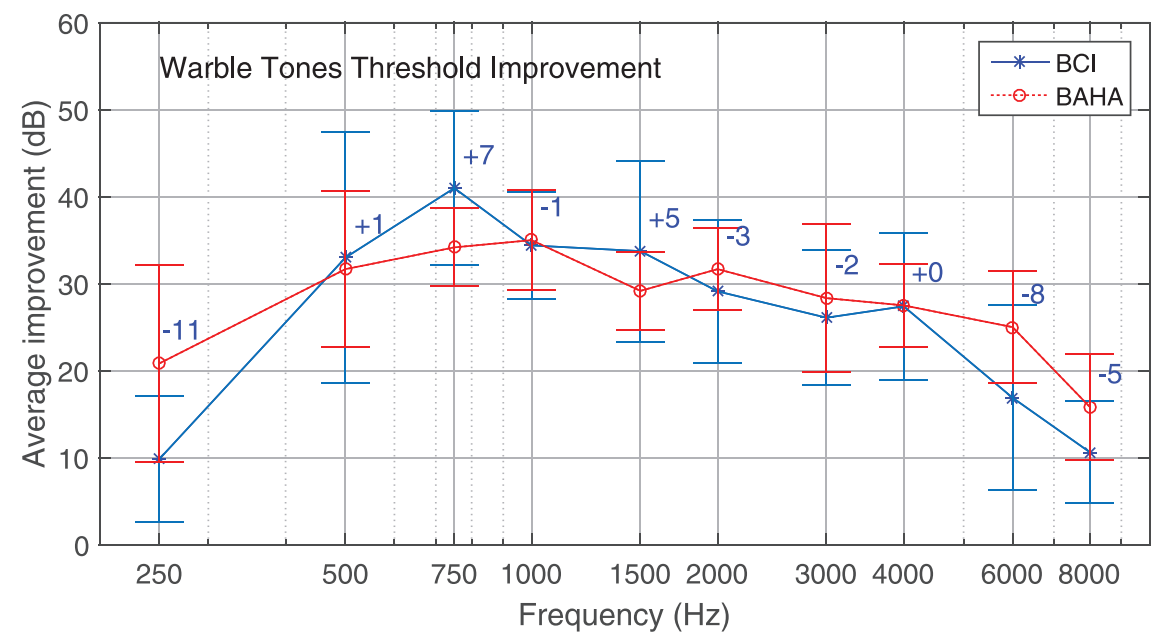

FIG. 1. Mean value and standard deviation in warble tone threshold improvement for the 2 different test groups. Blue numbers represent the difference between the mean improvement given by the $\mathrm{BCl}$ and the $\mathrm{BAHA}$ at specific frequencies. 

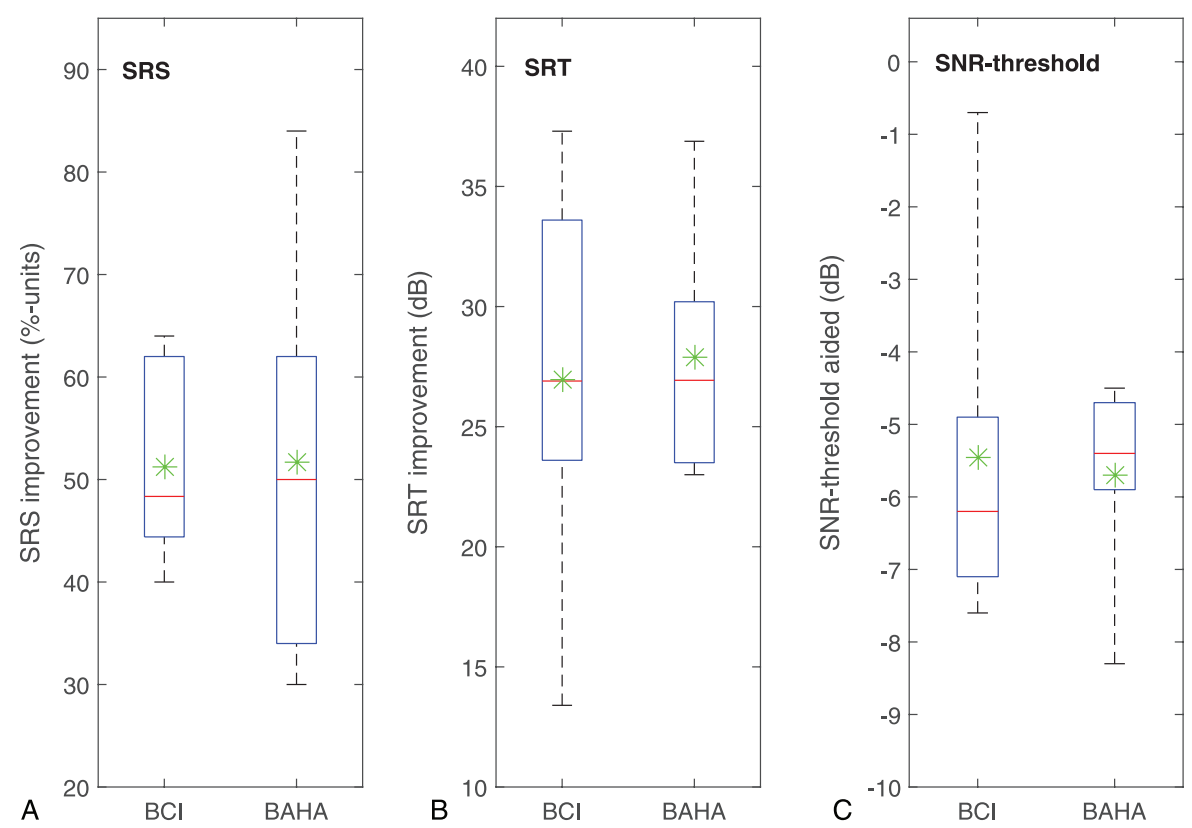

FIG. 2. Box plot of $(A)$ SRS improvement, $(B)$ SRT improvement, $(C)$ SNR threshold in aided condition in the 2 groups of patients (BCI, BAHA). SRS and SRT are expressed as an improvement from unaided to aided condition, SNR is expressed as absolute value measured only for aided condition. Median values are indicated by the red line, mean values with a green star, 25th and 75 th percentiles are included in the boxes.

unaided condition rather than absolute scores. Sex and age match had to be neglected in one case each: this is considered to have limited effect on the outcome of the audiometric measurements as hearing characteristics are considered to be the most influencing factors. Regarding the patient-related outcomes, these aspects could have a higher impact as the utilized questionnaires investigate everyday life situations that patients with different age and lifestyle could face more or less often and could evaluate in a different way. On the other hand, both questionnaires are specifically designed to target a wide range of patients and to evaluate the impact of the device regardless of personal details and habits.

\section{Audiometric Measurement Outcomes}

Speech tests were performed with the aim of testing different hearing situations which are meant to be a simplification of everyday life experience: speech in quiet at threshold level (SRT), speech at a conversational level in low (SRS), and high-noise environment (SNRthreshold). Hearing aid devices are usually equipped with automatic functions to improve directionality detection, feedback control, noise reduction, and a choice of different settings that the users can choose depending on their preferences. In this study, no automatic functions were activated to give a more authentic evaluation of the device potential, not being influenced by software add-ons. By allowing the use of such functionalities, the performance is expected to increase in a similar extent for both devices when adequate settings are employed.

Speech in noise testing is an important part of the study, since hearing aid users experience more difficulty in understanding speech in noise compared with normal

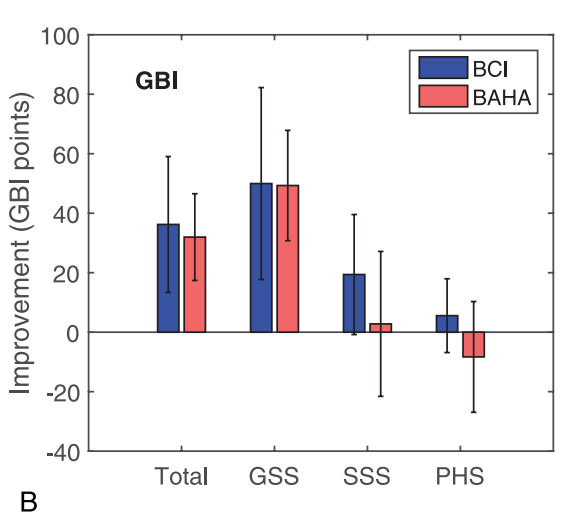

FIG. 3. Results from $A P H A B$ and $G B I$ questionnaires in all their subscales for the two groups of patients (BCI, BAHA). For $A P H A B$ : EC, BN, RV, AV. For GBI: general subscale score (GSS), social support subscale (SSS), PHS. 
hearing subjects and are therefore likely to require a higher SNR. Hearing in noise ability is not linearly related to the amount of hearing loss in PTA (31), and variability in SNR threshold in subjects with similar PTA loss was shown to be very high (32). The more negative SNR threshold, the more noise the patients can accept and still discriminate speech. On a linear scale, SNR threshold of $-6 \mathrm{~dB}$ means that when the level of noise is twice the level of speech, half of the words can still be correctly detected. A study by Smeds et al. (33) estimated that very few of the situations commonly encountered by hearing aid users in real-life sound environments present a negative SNR. In this scenario, an SNR threshold of around $-5 \mathrm{~dB}$, as resulted from measurements on both patient groups, shows that the devices provide an adequate rehabilitation to interact in noisy environments. However, measurements were performed with frontal speakers and results may differ for other spatial configurations of sound sources.

\section{Patient-related Outcomes}

Similar results obtained by both groups in all audiological measurements confirm the hypothesis that the BCI has the potential to offer a satisfying rehabilitation for indicated patients and that it can compete with percutaneous BCDs in terms of adequacy of amplification. It is nevertheless important to consider that measurements carried out in laboratories are just a simulation of real-life environment, so it is important not to limit the analysis to tone and speech audiometry, but to relate it directly to the user's experience. This was done with two questionnaires where common hearing situations were evaluated by the patients. Looking at the APHAB subscales EC, BN, and RV, results show satisfaction from both groups of patients, with slightly superior performance of the BCI, especially in favorable listening environments (EC subscale). AV subscale deals with unpleasant sounds, such as fire alarm, traffic noise, or water flush. A negative score seems to relate to the common complaints that hearing aids cause some environmental sound to become inappropriately loud (34), especially when high frequencies are over-amplified. Johnson et al. (35) found an improvement in AV scores from linear conventional hearing aids to compression technology and they hypothesize a positive contribution from noise reduction algorithms as well. This could somehow relate to the slightly lower result of $\mathrm{BCI}$ as for this newly developed BCD, automatic function algorithms are still to be implemented. The other questionnaire used in this study (GBI) was developed specifically for otorhinolaryngological interventions. In a study by Robinson et al. (28), it was shown that results from GBI are directly linked to effectiveness of middle ear surgery aimed at improving hearing: little improvement of hearing resulted in no benefit reported by the questionnaire, while an improvement of more than $15 \mathrm{~dB}$ in $\mathrm{PTA}_{4}$ resulted in positive benefit reported by the patients ( +34 points on the average). The results obtained from this comparative study are consistent with the literature, with an average $\mathrm{PTA}_{4}$ improvement of +31.0 and $+31.5 \mathrm{~dB}$ resulting in +36 and +32 points in total GBI score for the BCI and the BAHA group, respectively. PHS is a subscale of GBI dealing with physical aspect of health condition, investigating how the patient's status changed based on how the device implantation influenced the frequency of visits to doctor, medicine consumption, and inflammation occurrence. BCI patients reported a positive improvement, while negative benefit was reported by the BAHA group. This difference might be related to complications that the BAHA users sometimes face due to the skin-penetrating screw and uncomfortable wear of the device. The BCI, with its active transcutaneous technology and low profile, aims at solving such problems and encouraging response emerged by the questionnaire results. On the other hand, relatively short-term outcomes are compared with longterm evaluation in this study and an enthusiasm bias potentially affecting shorter-term users might have played a role in favor of the BCI. In this regard, literature results are equivocal. Self-reported outcomes seem however to stabilize within the first year of usage of the device and short-term evaluation seems to correlate well with long-term results $(36,37)$.

The study needs to be extended to more participants to have improved statistical power and possibly to include some intrasubject variability measurements with repeated tests on the same subject to investigate the statistical reliability and robustness of the measurements.

\section{CONCLUSION}

An early observational comparative study was performed to investigate the rehabilitation provided by the active transcutaneous BCI compared with established percutaneous devices. Six patients wearing BCI and six BAHA users were included in the study with a matched-pairs design. Audiological measurements were performed in both groups with no statistically significant differences detected in the results. Questionnaires were also used to assess the general health condition, benefit, and satisfaction level of patients. The outcome was slightly superior for $\mathrm{BCI}$ in all subscales.

Results from this study confirm the initial hypothesis: the BCI has the potential to provide as good rehabilitation as percutaneous devices for indicated patients.

Acknowledgments: This study was supported by Hearing Research Foundation and Swedish Research Council.

\section{REFERENCES}

1. Powell RH, Burrell SP, Cooper HR, Proops DW. The Birmingham bone anchored hearing aid programme: Pediatric experience and results. J Laryngol Otol Suppl 1996;21:21-9.

2. Dun CA, Faber HT, de Wolf MJ, Cremers CW, Hol MK. An overview of different systems: The bone-anchored hearing aid. $A d v$ Otorhinolaryngol 2011;71:22-31.

3. Pfiffner F, Caversaccio MD, Kompis M. Audiological results with BAHA in conductive and mixed hearing loss. Adv Otorhinolaryngol 2011;71:73-83. 
4. Dun CA, Faber HT, de Wolf MJ, Mylanus EA, Cremers CW, Hol MK. Assessment of more than 1,000 implanted percutaneous bone conduction devices: Skin reactions and implant survival. Otol Neurotol 2012;33:192-8.

5. van de Berg R, Stokroos RJ, Hof JR, Chenault MN. Bone-anchored hearing aid: A comparison of surgical techniques. Otol Neurotol 2010;31:129-35.

6. Husseman J, Szudek J, Monksfield P, Power D, O’Leary S, Briggs R. Simplified bone-anchored hearing aid insertion using a linear incision without soft tissue reduction. J Laryngol Otol 2013;127 (suppl 2):S33-8.

7. Reinfeldt S, Håkansson $B$, Taghavi $H$, Eeg-Olofsson $M$. New developments in bone-conduction hearing implants: A review. Med Devices (Auckl) 2015;8:79-93.

8. Hakansson B, Reinfeldt S, Eeg-Olofsson M, et al. A novel bone conduction implant (BCI): Engineering aspects and pre-clinical studies. Int $J$ Audiol 2010;49:203-15.

9. Reinfeldt S, Håkansson B, Taghavi H, Eeg-Olofsson M. Bone conduction hearing sensitivity in normal-hearing subjects: Transcutaneous stimulation at BAHA vs BCI position. Int $J$ Audiol 2014;53:360-9.

10. Eeg-Olofsson M, Stenfelt S, Tjellström A, Granström G. Transmission of bone-conducted sound in the human skull measured by cochlear vibrations. Int J Audiol 2008;47:761-9.

11. Taghavi $H$, Håkansson $B$, Reinfeldt $S$, et al. Technical design of a new bone conduction implant (BCI) system. Int J Audiol 2015;54:736-44.

12. Cremers CW, Snik FM, Beynon AJ. Hearing with the bone-anchored hearing aid (BAHA, HC 200) compared to a conventional boneconduction hearing aid. Clin Otolaryngol Allied Sci 1992;17:275-9.

13. Carlsson P, Håkansson B, Rosenhall U, Tjellström A. A speech-tonoise ratio test with the bone-anchored hearing aid: A comparative study. Otolaryngol Head Neck Surg 1986;94:421-6.

14. Busch S, Giere T, Lenarz T, Maier H. Comparison of audiologic results and patient satisfaction for two osseointegrated bone conduction devices: Results of a prospective study. Otol Neurotol 2015;36:842-8.

15. Finbow J, Bance M, Aiken S, Gulliver M, Verge J, Caissie R. A comparison between wireless CROS and bone-anchored hearing devices for single-sided deafness: A pilot study. Otol Neurotol 2015;36:819-25.

16. Wade PS, Halik JJ, Chasin M. Bone conduction implants: Transcutaneous vs. percutaneous. Otolaryngol Head Neck Surg 1992;106:68-74.

17. Iseri M, Orhan KS, Tuncer U, et al. Transcutaneous bone-anchored hearing aids versus percutaneous ones: Multicenter comparative clinical study. Otol Neurotol 2015;36:849-53.

18. Reinfeldt S, Håkansson B, Taghavi H, Fredén Jansson KJ, EegOlofsson M. The bone conduction implant: Clinical results of the first six patients. Int J Audiol 2015;54:408-16.
19. Eeg-Olofsson $\mathrm{M}$, Håkansson $\mathrm{B}$, Reinfeldt $\mathrm{S}$, et al. The bone conduction implant-first implantation, surgical and audiologic aspects. Otol Neurotol 2014;35:679-85.

20. Stenfelt S, Reinfeldt S. A model of the occlusion effect with boneconducted stimulation. Int J Audiol 2007;46:595-608.

21. American Speech-Language-Hearing Association. Guidelines for manual pure-tone threshold audiometry. 2005.

22. ISO, ISO 8253-1. Acoustics-Audiometric test methods: Part 1: Pure-tone air and bone conduction audiometry. 2010.

23. ISO, ISO 8253-3. Acoustics-Audiometric test methods: Part 3: Speech audiometry. 2012.

24. Hagerman B. Sentences for testing speech intelligibility in noise. Scand Audiol 1982;11:79-87.

25. Hagerman B. Efficiency of speech audiometry and other tests. Br J Audiol 1993;27:423-5.

26. Hagerman B, Kinnefors C. Efficient adaptive methods for measuring speech reception threshold in quiet and in noise. Scand Audiol 1995;24:71-7.

27. Cox RM, Alexander GC. The abbreviated profile of hearing aid benefit. Ear Hear 1995;16:176-86.

28. Robinson K, Gatehouse S, Browning GG. Measuring patient benefit from otorhinolaryngological surgery and therapy. Ann Otol Rhinol Laryngol 1996;105:415-22.

29. Hol MK, Nelissen RC, Agterberg MJ, Cremers CW, Snik AF. Comparison between a new implantable transcutaneous bone conductor and percutaneous bone-conduction hearing implant. Otol Neurotol 2013;34:1071-5.

30. Gerdes T, Salcher RB, Schwab B, Lenarz T, Maier H. Comparison of audiological results between a transcutaneous and a percutaneous bone conduction instrument in conductive hearing loss. Otol Neurotol 2016;37:685-91.

31. Killion MC. The SIN report: Circuits haven't solved the hearing-innoise problem. Hear $J$ 1997;50:28-30. 32, 34.

32. Killion MC, Niquette PA. What can the pure-tone audiogram tell us about patient's SNR loss? Hear J 2000;53:46-8.

33. Smeds K, Wolters F, Rung M. Estimation of signal-to-noise ratios in realistic sound scenarios. J Am Acad Audiol 2015;26: 183-96.

34. Cox R. Administration and application of the APHAB. Hear $J$ 1997;50:32-48.

35. Johnson JA, Cox RM, Alexander GC. Development of APHAB norms for WDRC hearing aids and comparisons with original norms. Ear Hear 2010;31:47-55.

36. Taylor KS. Self-perceived and audiometric evaluations of hearing aid benefit in the elderly. Ear Hear 1993;14:390-4.

37. Spirakis GJ. Long term stability of self reported hearing aid benefit in adults. Graduate Theses and Dissertations. 2002. Available at: http://scholarcommons.us£edu/etd/2950. Accessed June 14, 2016. 